\title{
Discovery of a red supergiant counterpart to RX J004722.4-252051, a ULX in NGC 253
}

\author{
M. Heida, ${ }^{1,2 \star}$ M. A. P. Torres, ${ }^{1,2,3}$ P. G. Jonker, ${ }^{1,2 \star ~ M . ~ S e r v i l l a t, ~}{ }^{4,5}$ S. Repetto, ${ }^{2}$ \\ T. P. Roberts, ${ }^{6}$ D. J. Walton, ${ }^{7}$ D.-S. Moon ${ }^{8}$ and F. A. Harrison ${ }^{7}$ \\ ${ }^{1}$ SRON Netherlands Institute for Space Research, Sorbonnelaan 2, NL-3584 CA Utrecht, the Netherlands \\ ${ }^{2}$ Department of Astrophysics/IMAPP, Radboud University Nijmegen, PO Box 9010, NL-6500 GL Nijmegen, the Netherlands \\ ${ }^{3}$ European Southern Observatory, Alonso de Cordova 3107, Casilla 19001, Vitacura, Santiago 19, Chile \\ ${ }^{4}$ Laboratoire AIM (CEA/Irfu/SAp, CNRS/INSU, Université Paris Diderot), CEA Saclay, F-91191 Gif-sur-Yvette, France \\ ${ }^{5}$ Harvard-Smithsonian Center for Astrophysics, 60 Garden Street, Cambridge, MA 02138, USA \\ ${ }^{6}$ Department of Physics, University of Durham, South Road, Durham DH1 3LE, UK \\ ${ }^{7}$ Space Radiation Laboratory, California Institute of Technology, Pasadena, CA 91125, USA \\ ${ }^{8}$ Department of Astronomy and Astrophysics, University of Toronto, Toronto, ON M5S 3H4, Canada
}

Accepted 2015 August 8. Received 2015 July 10; in original form 2015 April 20

\begin{abstract}
We present two epochs of near-infrared spectroscopy of the candidate red supergiant counterpart to RX J004722.4-252051, a ULX in NGC 253. We measure radial velocities of the object and its approximate spectral type by cross-correlating our spectra with those of known red supergiants. Our VLT/X-shooter spectrum is best matched by that of early M-type supergiants, confirming the red supergiant nature of the candidate counterpart. The radial velocity of the spectrum, taken on 2014 August 23, is $417 \pm 4 \mathrm{~km} \mathrm{~s}^{-1}$. This is consistent with the radial velocity measured in our spectrum taken with Magellan/MMIRS on 2013 June 28, of $410 \pm 70 \mathrm{~km} \mathrm{~s}^{-1}$, although the large error on the latter implies that a radial velocity shift expected for a black hole of tens of $\mathrm{M}_{\odot}$ can easily be hidden. Using nebular emission lines we find that the radial velocity due to the rotation of NGC 253 is $351 \pm 4 \mathrm{~km} \mathrm{~s}^{-1}$ at the position of the ULX. Thus the radial velocity of the counterpart confirms that the source is located in NGC 253, but also shows an offset with respect to the local bulk motion of the galaxy of $66 \pm 6 \mathrm{~km} \mathrm{~s}^{-1}$. We argue that the most likely origin for this displacement lies either in a SN kick, requiring a system containing a $\gtrsim 50 \mathrm{M}_{\odot}$ black hole, and/or in orbital radial velocity variations in the ULX binary system, requiring a $\gtrsim 100 \mathrm{M} \odot$ black hole. We therefore conclude that RX J004722.4-252051 is a strong candidate for a ULX containing a massive stellar black hole.
\end{abstract}

Key words: supergiants - infrared: stars $-\mathrm{X}$-rays: binaries $-\mathrm{X}$-rays: individual: $\mathrm{RX}$ J004722.4-252051.

\section{INTRODUCTION}

Ultraluminous X-ray sources (ULXs) are defined as off-nuclear $\mathrm{X}$-ray sources with a luminosity greater than the Eddington luminosity of a $10 \mathrm{M}_{\odot}$ black hole $(\mathrm{BH})$; in practice a limit for the $0.3-10.0 \mathrm{keV}$ luminosity $\geq 10^{39} \mathrm{erg} \mathrm{s}^{-1}$ is used (for a review see e.g. Feng \& Soria 2011). The two main scenarios to explain these high luminosities are (1) super-Eddington accretion on to stellar mass BHs or neutron stars (Begelman 2002) and (2) sub-Eddington accretion on to intermediate mass BHs (IMBHs; Colbert \& Mushotzky 1999).

\footnotetext{
^E-mail: m.heida@astro.ru.nl (MH); p.jonker@ sron.nl (PGJ)
}

A growing body of evidence indicates that the group of sources we call ULXs may contain objects of both kinds. A recent discovery of X-ray pulses from M82 ULX-2 proves that the accretor in the system is a neutron star (Bachetti et al. 2014). Yet this ULX sometimes reaches an X-ray luminosity of $1.8 \times 10^{40} \mathrm{erg} \mathrm{s}^{-1}$, which is clearly orders of magnitude above its Eddington limit. Liu et al. (2013) use optical spectroscopic observations of the donor star of M101 ULX1 and Motch et al. (2014) use optical spectroscopic and photometric observations of the donor star of NGC 7793 P13 to calculate a dynamical limit on the $\mathrm{BH}$ masses in these systems. In both cases they find the accretor is most likely a stellar mass BH. These recent discoveries support the suggestion of Gladstone, Roberts \& Done (2009) and others that the peculiar X-ray spectral state, seen in several ULXs with high-quality X-ray data (e.g. Bachetti et al. 2013; Sutton, Roberts \& Middleton 2013; Pintore et al. 2014), is 
an 'ultraluminous' state and that many ULXs are stellar mass BHs emitting above their Eddington limit.

However, the brightest ULXs, with luminosities above $10^{41}$ $\operatorname{erg~} \mathrm{s}^{-1}$ (also known as hyperluminous X-ray sources [HLXs]), are still good candidates to host IMBHs (e.g. Farrell et al. 2009; Jonker et al. 2010; Sutton et al. 2012). The brightest known ULX and strongest candidate for hosting an IMBH is ESO 243-49 HLX1, with an estimated BH mass of $\sim 20000 \mathrm{M}_{\odot}$ (Farrell et al. 2009). M82 ULX-1 and NGC 2276-3c are two other ULXs that are candidate IMBHs (Pasham, Strohmayer \& Mushotzky 2014; Mezcua et al. 2015).

The most reliable, model-independent method to measure the mass of the accretors in ULXs is through dynamical mass measurements. The first attempts to do so (Roberts et al. 2011; Liu, Orosz \& Bregman 2012) used optical emission lines from the accretion discs in these systems. However, these do not show periodic motion. A more promising approach is to measure the radial velocity curve of the donor star, as has been done for several Galactic X-ray binaries (e.g. McClintock \& Remillard 1986).

The optical spectra of many ULX counterparts do not show stellar features, as they are dominated by emission from the accretion disc (cf. Grisé et al. 2012; Tao et al. 2012; Sutton, Done \& Roberts 2014). Some ULXs may however have red supergiant (RSG) donor stars. Since RSGs are intrinsically very bright in the near-infrared (NIR) it is possible to measure their radial velocities spectroscopically out to $\sim 10 \mathrm{Mpc}$. An additional advantage is that in the NIR the contribution from the accretion disc is lower than in the optical regime. Also, because of the large orbital separations in such systems, irradiation of the donor star is not expected to play an important role (Copperwheat et al. 2007). Therefore, we performed a photometric survey of nearby $(D<10 \mathrm{Mpc})$ ULXs in the NIR to search for ULXs with RSG donor stars (Heida et al. 2014).

In this paper, we present the results of NIR spectroscopic followup of the ULX with the brightest candidate NIR counterpart $(K s=17.2 \pm 0.5)$ in the sample presented in Heida et al. (2014): RX J004722.4-252051 in NGC 253 (hereafter J0047), at a distance of 3.4 Mpc (Dalcanton et al. 2009). The counterpart is also listed in the catalogue by Bailin et al. (2011) (source ID 47620), with $V=21.58$ and $I=19.96$. Given this $V$-band magnitude and the $K s$-band magnitude from Heida et al. (2014), we derive that the $V$ $-K=4.4 \pm 0.5$ colour is consistent with that of an RSG, assuming both the $V$ and $K$ magnitudes do not vary strongly in time.

The ULX has been studied in X-rays by for example Barnard (2010) (NGC 253 ULX1), Sutton et al. (2013) (NGC 253 XMM2), Pintore et al. (2014) (NGC 253 X-1) and Wik et al. (2014) (source 7). It is not a particularly bright ULX, with a peak luminosity in the $0.3-10 \mathrm{keV}$ band of $2.4 \times 10^{39} \mathrm{erg} \mathrm{s}^{-1}$ (Pintore et al. 2014), and it has been observed to drop well below the ULX threshold in other observations. Additionally, it shows unusually high variability for a disc-like object (Sutton et al. 2013). The spectrum was classified by Sutton et al. (2013) as a 'broadened disc', an ultraluminous state associated with sources accreting around their Eddington limit (Gladstone et al. 2009). This would imply a mass of $\lesssim 20 \mathrm{M}_{\odot}$ for the $\mathrm{BH}$ in this system.

\section{OBSERVATIONS AND DATA REDUCTION}

We obtained two spectra of J0047 separated by about one year: one with the MMT and Magellan Infrared Spectrograph (MMIRS; McLeod et al. 2012) on the Magellan Clay telescope at Las Campanas Observatory, and the other with X-shooter (Vernet et al. 2011) on the Very Large Telescope (VLT) UT3 at Cerro Paranal.

\section{Magellan/MMIRS}

The MMIRS spectrum (proposal ID 2013A-CFA-3) was taken on the night of 2013, June 28, with a 2-pixel ( $0.4 \mathrm{arcsec})$ slit, the HK grism and the HK filter. This setup yields a spectrum from 1.25 to $2.45 \mu \mathrm{m}$, with an average spectral resolution $\left(\frac{\Delta \lambda}{\lambda}\right)$ of $R \approx 1400$. We used an ABBA nodding pattern with a dither offset step of 20 arcsec. We obtained 16 spectra with exposure times of $300 \mathrm{~s}$ each, for a total time on source of $4800 \mathrm{~s}$. The data were reduced through the CfA MMIRS pipeline (v0.9.7RC7-20140301; Chilingarian et al. 2013). This pipeline subtracts the two exposures of one dither pair, performs a flat-field and residual sky correction and rectifies the $2 \mathrm{D}$ spectra. The spectra are wavelength-calibrated using the positions of telluric $\mathrm{OH}$-emission lines; the rms of the residuals of the wavelength solution is $0.6 \AA$. The dither pairs are then co-added and telluric absorption lines are removed using a telluric standard star observed close in time to and at similar airmass as the target. These corrected 2D spectra still have both a positive and a negative trace of the object. We use the Starlink program FIGARo for the further data reduction steps: use the optimal extraction algorithm by Horne (1986) to extract the spectrum of the positive trace from the pipeline-reduced 2D spectrum, the spectrum of the negative trace from the inverted 2D spectrum, and then add the two to obtain the final spectrum. The nodding pattern unfortunately projected the positive trace of J0047 on top of the fainter, extended negative trace of another region of NGC 253 . We use a narrow extraction region for the positive trace to minimize the effects of this configuration. We correct the wavelength calibration of the MMIRS pipeline (that is done using wavelengths in vacuum) to match the calibration of our other spectra (that are calibrated using wavelengths in air).

\section{VLT/X-shooter}

The X-shooter spectrum (programme ID 093.D-0256) was taken in service mode on the night of 2014, August 23. X-shooter has three spectroscopic arms that operate simultaneously, providing spectral coverage from the near UV to the near-IR. We used slit widths of 0.8 arcsec in the UVB arm, 0.7 arcsec in the VIS arm and 0.6 arcsec in the NIR arm, resulting in resolutions of 6200, 10600 and 7780, respectively. We used X-shooter in nodding mode, with an ABBA nodding pattern and a nod throw of 5 arcsec. The integration times for the UVB, VIS and NIR arms were 260, 210 and $285 \mathrm{~s}$, respectively. The total exposure times for the three arms are 2600, 2100and $2850 \mathrm{~s}$.

In the NIR arm, the signal-to-noise ratio $(\mathrm{S} / \mathrm{N})$ per spectral resolution element in the $J$ and $H$ bands is $\sim 10$ in regions that are not affected by atmospheric emission lines. Because the throughput of X-shooter drops rapidly towards the $K$ band, that part of the spectrum is not useful.

We process the NIR observations of the ULX counterpart and a telluric standard star at similar airmass observed close in time to the target with the X-shooter pipeline workflow in the REFLEX environment (Freudling et al. 2013). The pipeline produces flatfielded, sky-subtracted, rectified, wavelength- and flux-calibrated 1-D and 2D spectra. The rms amplitude of the residuals of the wavelength solution is $0.1 \AA$. The wavelength calibration is done with arcs that are taken during the day, not at the same airmass as the observations. This can introduce a small offset with respect to the real wavelength solution. We check this by comparing the positions of sky emission lines to the line list of Rousselot et al. (2000) and find an offset of $\approx 0.3 \AA$, that we correct for. 
We then use the SPEXTOOLS-task XTELLCOR_GENERAL (Vacca, Cushing \& Rayner 2003) to correct the spectrum for telluric absorption features.

The $H$ band spectra of RSGs contain several strong absorption edges from $\mathrm{CO}$ and lines from neutral metals that can be used to derive the effective temperature of the star.The $J$ band also contains absorption lines from neutral metals such as Fe, Ti and $\mathrm{Mg}$ that can be used to derive abundances, but they are not very sensitive to temperature (Davies, Kudritzki \& Figer 2010). We have verified that these absorption lines are present in the $J$ band, but in the remainder of this paper we focus our analysis on the $H$ band.

In the data from the UVB and VIS arms the $\mathrm{S} / \mathrm{N}$ of the counterpart is very low $(<1)$. However, there are spatially extended emission lines visible that appear centred on a source at $\sim 5$ arcsec East from the ULX counterpart (not detectable in the NIR arm) that is present in one of the nod positions (the ' $\mathrm{B}$ ' position). From inspection of archival VLT/FORS1 $R$ - and $I$-band images taken in the night of 2004, July 23 we know that there is a blue $(V-I=0$; Bailin et al. 2011) source that lies partially in the slits at that position. The presence of this source makes it impossible to process the data in nodding mode. We follow the $\mathrm{X}$-shooter pipeline manual and use the $x s h \_w k f \_s t a r e . o c a$ file to process the data in stare mode. We extract the emission line spectrum at the position of the counterpart from the exposures without the blue source in the slit (the 'A' position), and at a position close to the blue source from the exposures in the ' $\mathrm{B}$ ' nod position. For each exposure, the pipeline produces biassubtracted, flat-fielded, sky-subtracted, rectified, wavelength- and flux-calibrated 1-D and 2D spectra. It also produces $1 \mathrm{D}$ and 2D spectra of the subtracted sky emission. For the VIS arm, where there are many strong sky emission lines, we use these sky lines to check the wavelength calibration and find no systematic offset. The rms amplitude of the residuals of the wavelength solution for both the VIS and UVB spectra is $0.06 \AA$.

\section{ANALYSIS AND RESULTS}

\subsection{Cross-correlation analysis}

We are primarily interested in two properties of the candidate counterpart: its spectral type, to give us an indication of the temperature and (via the observed absolute magnitude) the radius of the star, and its radial velocity to confirm that it is a donor star in a binary that belongs to NGC 253 and is not a foreground or background object. Therefore, we cross-correlate our X-shooter spectrum with high S/N spectra of known RSGs of different spectral types (ranging from $\mathrm{K} 1$ to M4.5) and metallicities.

The set of RSG spectra that we use contains one Galactic RSG, CD-60 3621, whose heliocentric radial velocity is $-17.7 \pm 0.4 \mathrm{~km} \mathrm{~s}^{-1}$ (Mermilliod, Mayor \& Udry 2008; however, as their individual radial velocity measurements of the star show intrinsic variations from -16.0 to $-19.0 \mathrm{~km} \mathrm{~s}^{-1}$, we adopt a larger uncertainty of $3 \mathrm{~km} \mathrm{~s}^{-1}$. All uncertainties quoted in this paper are $1 \sigma$ errors). Its spectral type is M1.5 I (Levesque et al. 2005). This star was observed with X-shooter as part of the X-shooter Spectral Library on the night of 2010, February 3 (see Chen et al. 2014 for a description of the library). We retrieved the pipeline-reduced spectrum from the ESO phase 3 archive. The spectrum has been wavelength calibrated, but not corrected for telluric absorption. The observations were done with the 0.6 arcsec slit in the NIR-arm, so the resolution of the spectrum is the same as for our X-shooter spectrum (observations for both J0047 and CD-60 3621 were done with seeing $>0.6$ arcsec, so the resolution is set by the slit width).
The other RSGs in our comparison sample are those described by Davies et al. (2013). They observed 19 RSGs of a range of spectral types in the LMC and SMC with X-shooter. These spectra have been corrected for telluric absorption, but their radial velocities are not as accurately known as that of CD-60 3621. Since these observations were done with a wide $(5 \operatorname{arcsec})$ slit, they have a lower resolution than our $\mathrm{X}$-shooter spectrum $(R \approx 5000)$.

We use the program MOLLY for the analysis of the data. After reading the spectra into MOLLY, we first use $h f i x$ to calculate and subsequently apply heliocentric corrections. We prepare the spectra for cross-correlation following the recommendation in the MOLLY user guide, ${ }^{1}$ first normalizing with a second-order polynomial fit and then subtracting a fourth-order spline fit to the normalized spectra. We resample the spectra to a common velocity spacing (10.85 $\mathrm{km} \mathrm{s}^{-1} \mathrm{pix}^{-1}$ for our X-shooter spectrum, $118.4 \mathrm{~km} \mathrm{~s}^{-1}$ $\mathrm{pix}^{-1}$ for our MMIRS spectrum) with vbin and then run xcor to calculate the cross-correlation functions of J0047 with the comparison sample. xcor also calculates the radial velocity offset from the cross-correlation function by fitting a parabola to the three peak pixels. This is a generally robust estimate but it underestimates the uncertainty on the radial velocity. To get a better estimate of the uncertainty we fit a polynomial plus Gaussian curve to the crosscorrelation functions using $m g$ fit. As a sanity check we also calculate the cross-correlation functions of CD-60 3621 with the LMC/SMC RSGs. The parts of the spectrum of J0047 that are most affected by noise from strong telluric emission lines are masked out during the cross-correlation.

The cross-correlation between the $H$ band $\mathrm{X}$-shooter spectrum of J0047 and CD-60 3621 yields a velocity shift of $434.4 \pm 1.0 \mathrm{~km} \mathrm{~s}^{-1}$. This uncertainty is the statistical error on the determination of the position of the peak of the cross-correlation function. The total uncertainty includes the uncertainty on the wavelength calibration of X-shooter ( $2 \mathrm{~km} \mathrm{~s}^{-1}$ for both spectra) and the uncertainty in the radial velocity of CD-60 $3621\left(3 \mathrm{~km} \mathrm{~s}^{-1}\right)$. Quadratically adding all these uncertainties and adding the radial velocity of CD-60 3621 gives a velocity shift of $417 \pm 4 \mathrm{~km} \mathrm{~s}^{-1}$. The cross-correlations of J0047 with the SMC/LMC sample yield consistent results.

We obtain the highest values of the cross-correlation function for CD-60 3621 and LMC-136042 (the latter is an M3-type RSG). In addition, following the procedure described by Origlia, Moorwood \& Oliva (1993), we measure the equivalent widths of the CO (1.62 $\mu \mathrm{m})$ bandhead and the $\mathrm{Si}$ I $(1.59 \mu \mathrm{m})$ absorption line using the light command in MOLLY (see Fig. 1 where parts of the spectra are plotted, shifted to zero radial velocity with respect to the heliocentric frame). The ratio of these lines is sensitive to the temperature of the star. We find a ratio of $\log \left(W_{1.62} / W_{1.59}\right)=0.15 \pm 0.1$, which is consistent with an effective temperature in the range 3000-4000 K [Förster Schreiber 2000; with the caveat that our spectrum has a higher resolution than those of Förster Schreiber (2000) and Origlia et al. (1993), and it is not known if this will affect the line ratio].

The cross-correlation functions for the SMC RSGs all have lower values than those for the LMC RSGs. From this we conclude that J0047 is an early M-type RSG, with a metallicity closer to that of the LMC and Milky Way RSGs than to that of those in the SMC, which have a lower metallicity. This is not unexpected, as abundance measurements of $\mathrm{H}$ II regions in NGC 253 yield values for $12+\log (\mathrm{O} / \mathrm{H})$ in the range 8.5-9.0 (Webster \& Smith 1983), comparable to abundance ratios found in $\mathrm{H}$ II regions in the Milky

\footnotetext{
${ }^{1}$ http://deneb.astro.warwick.ac.uk/phsaap/software/molly/html/USER GUIDE.html
} 

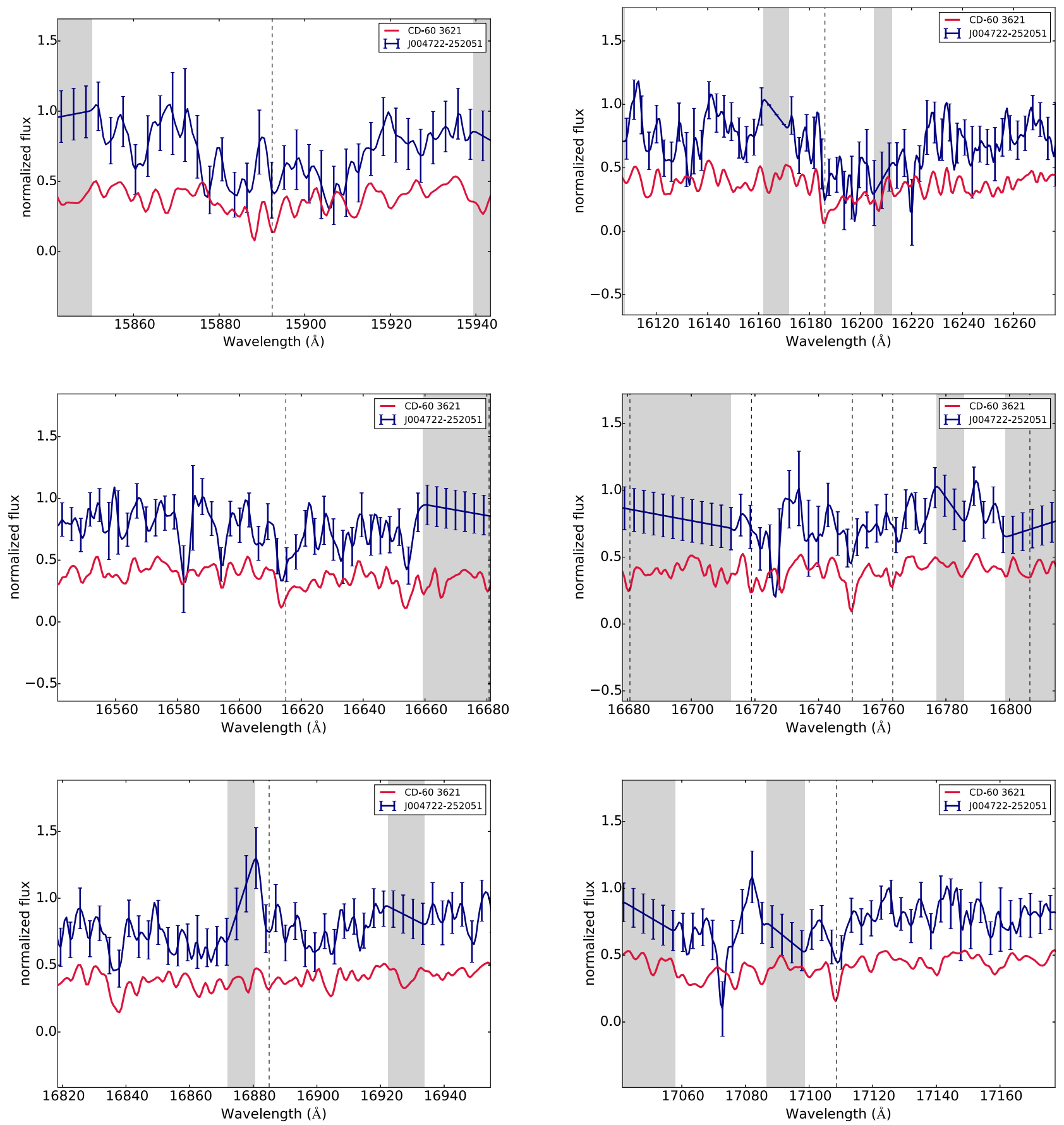

Figure 1. Sections of the $H$ band X-shooter spectrum of J0047 (upper, blue line) and the X-shooter spectrum of CD-60 3621 (lower, red line). Both spectra are normalized and smoothed to the resolution of X-shooter. For presentation purposes only, we interpolated the spectrum of J0047 over areas that are strongly affected by telluric emission (grey shaded areas). Error bars are plotted for every fifth data point. The spectrum of CD-60 3621 is shifted vertically by -0.5 . The J0047 spectrum is shifted by $-417 \mathrm{~km} \mathrm{~s}^{-1}$ and the CD-60 3621 spectrum is shifted by $17 \mathrm{~km} \mathrm{~s}^{-1}$. The dashed vertical lines indicate the positions of atomic and molecular lines (left to right, top to bottom: $\mathrm{Si}$ I $(1.59 \mu \mathrm{m}), \mathrm{CO}$ bandhead $(1.62 \mu \mathrm{m}), \mathrm{CO}$ bandhead $(1.66 \mu \mathrm{m}), \mathrm{Al}$ I triplet $(1.672,1.675,1.676 \mu \mathrm{m}), \mathrm{OH}$ $(1.69 \mu \mathrm{m}), \operatorname{Mg} \mathrm{I}(1.71 \mu \mathrm{m})$.

Way (e.g. Balser et al. 2011). Values found for $\mathrm{H}$ II regions in the LMC and SMC are $8.4 \pm 0.12$ and $7.98 \pm 0.09$, respectively (Pagel et al. 1978).

The Magellan/MMIRS spectrum of J0047, due to its much lower resolution and lower $\mathrm{S} / \mathrm{N}$, can not be used to constrain the spectral type of the RSG. Therefore, we cross-correlate this spectrum only with CD-60 3621, resampled to the velocity resolution of the MMIRS spectrum. We initially mask only those pixels that are strongly affected by residuals from telluric emission lines. This leaves 169 pixels (from a total of 463) for the cross-correlation. The peak of the cross-correlation function is found at a shift of $3.5 \pm 0.2$ pixels, or $410 \pm 20 \mathrm{~km} \mathrm{~s}^{-1}$. As a consistency check we also cross-correlate the spectra using only regions where we could visually identify absorption lines. This leaves us with 63 pixels. The 


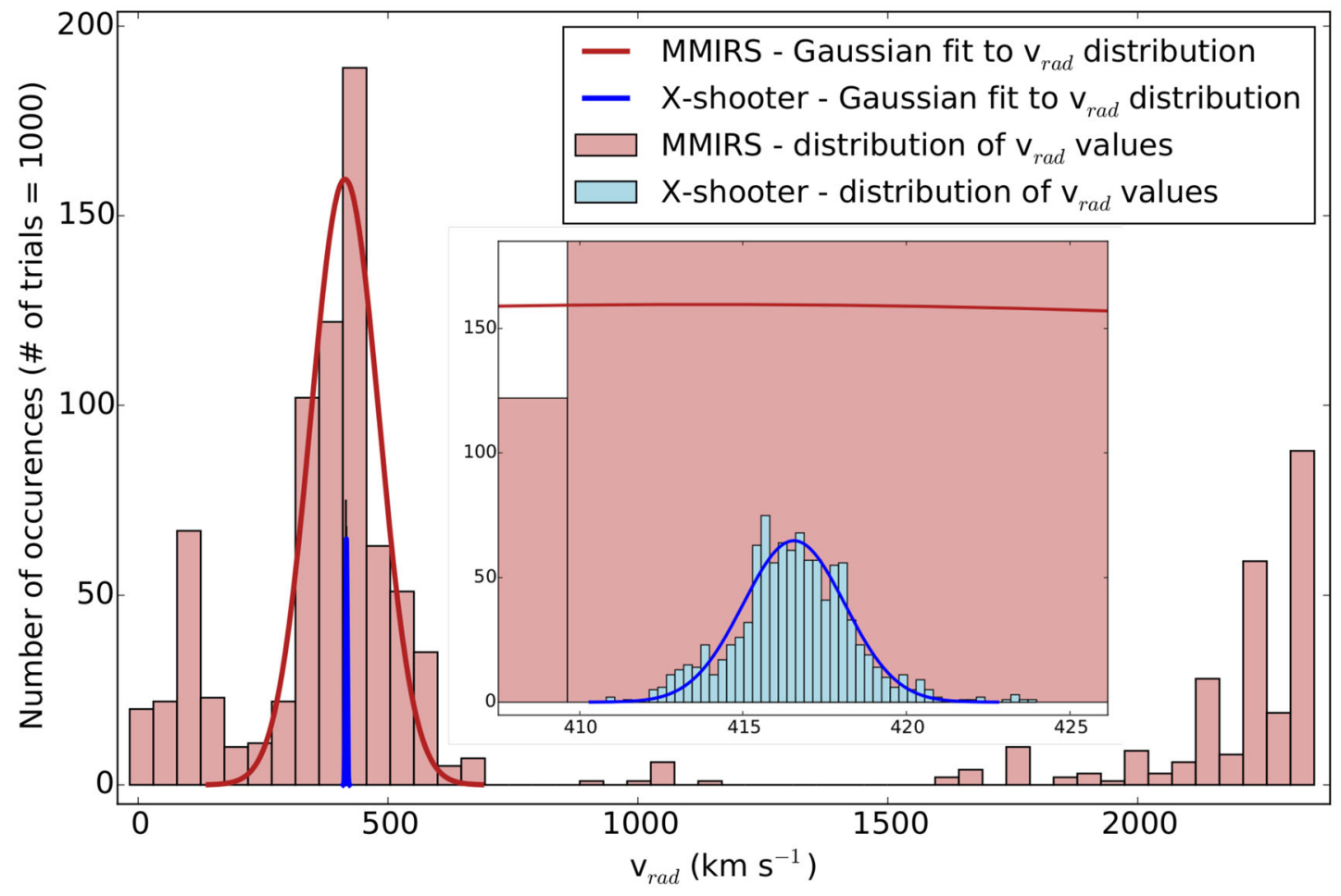

Figure 2. The histograms show the distribution of radial velocities obtained by cross-correlating 1000 bootstrapped copies of the MMIRS spectrum (red) and the X-shooter spectrum (blue) with CD-60 3621. The systemic velocity of CD-60 3621 of $17 \mathrm{~km} \mathrm{~s}^{-1}$ is already subtracted. The best-fitting Gaussian curves to the data are also plotted (red/blue lines). Inset is a zoom-in of the image around $415 \mathrm{~km} \mathrm{~s}^{-1}$ to show the X-shooter data more clearly.

shift found in this way is consistent with the first: $3.6 \pm 0.3$ pixels, or $430 \pm 30 \mathrm{~km} \mathrm{~s}^{-1}$. Correcting these shifts for the radial velocity of CD-60 3621 gives values of $400 \pm 20$ and $410 \pm 30 \mathrm{~km} \mathrm{~s}^{-1}$, respectively. However, the uncertainty on the position of the peak of these cross-correlation functions is calculated assuming they have a Gaussian profile. This is the case for the X-shooter data but not for the MMIRS data. Therefore, the error on the radial velocity of the MMIRS spectrum is underestimated.

To gain more insight in the uncertainties on the radial velocities, we perform a bootstrap analysis. We use the MOLLY command boot to produce 1000 resampled copies of the MMIRS and X-shooter spectra. We then cross-correlate these with the spectrum of CD-60 3621 using $x$ cor, and fit a Gaussian to the resulting distribution of radial velocities. The width of this Gaussian is a good measure of the uncertainty on the radial velocity. After correcting for the radial velocity of CD-60 3621 we find a value of $416.6 \pm 1.6 \mathrm{~km} \mathrm{~s}^{-1}$ for the $\mathrm{X}$-shooter spectrum. As we expected, this value is consistent with the one we obtained directly from the cross-correlation. For the MMIRS spectrum, we find a radial velocity of $410 \pm 70 \mathrm{~km} \mathrm{~s}^{-1}$ if we use the 169-pixel mask (see Fig. 2), and $410 \pm 30 \mathrm{~km} \mathrm{~s}^{-1}$ with the 63-pixel mask. We conservatively adopt that the radial velocity of J0047 as measured from the MMIRS spectrum is $410 \pm 70 \mathrm{~km} \mathrm{~s}^{-1}$.

\subsection{Nebular emission lines}

Although the UVB and VIS spectra have very weak continua (UVB: $\mathrm{S} / \mathrm{N}<1$, VIS: $\mathrm{S} / \mathrm{N} \approx 1$ ) there are several emission lines visible in the data. In the UVB data, these are the [O II] $\lambda \lambda 3727,3729$, $\mathrm{H} \beta$ and $[\mathrm{O}$ III] $\lambda \lambda 4956,5007$ lines. In the VIS spectrum we detect [N II] $\lambda 6548, \mathrm{H} \alpha,[\mathrm{N}$ II] $\lambda 6583$ and the [S II] $\lambda \lambda 6716,6731$ doublet. Inspection of the $2 \mathrm{D}$ spectra reveals that these lines do not fill the whole slit but are extended in the direction of - and peak around
- the bright source that dominates the unused nod position B (this source is located $\sim 5$ arcsec East of the RSG, but is not visible in our NIR image due to its blue colour; for the 1- and 2-D VIS spectra see Fig. 3). After reading the spectra of the single exposures into

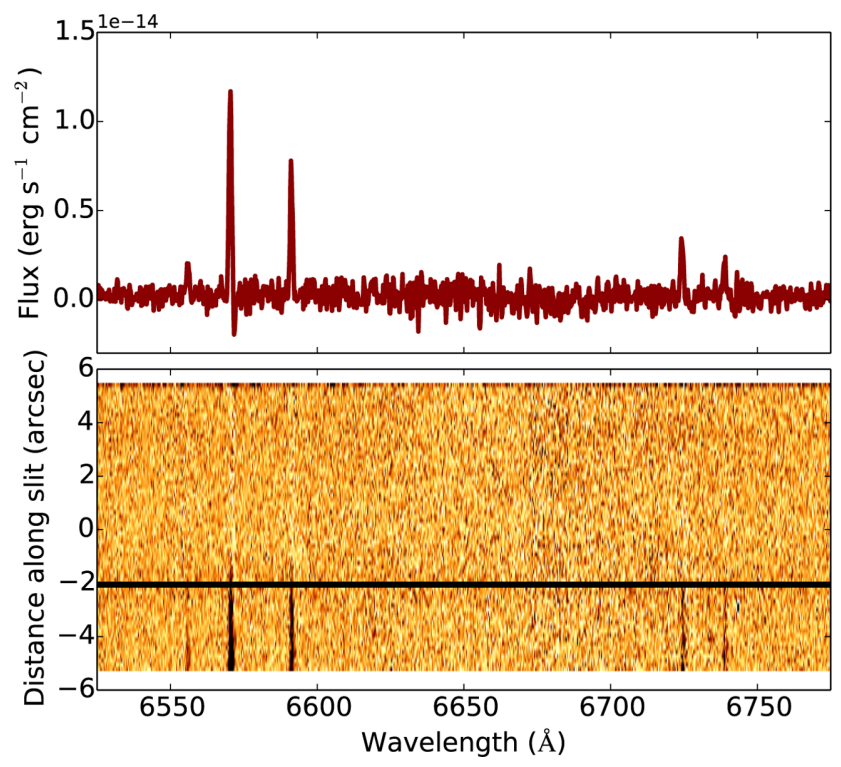

Figure 3. Part of the VIS spectrum with the $[\mathrm{N}$ II] $\lambda 6548, \mathrm{H} \alpha$, [N II] $\lambda 6583$ complex and the [S II] $\lambda \lambda 6716,6731$ doublet. Upper figure: the fluxcalibrated spectrum extracted at the position of the ULX. Lower figure: the 2-D image of the spectrum. Here it can clearly be seen that the emission lines are extended. The black horizontal line indicates the position of the RSG (not visible) on the detector. 

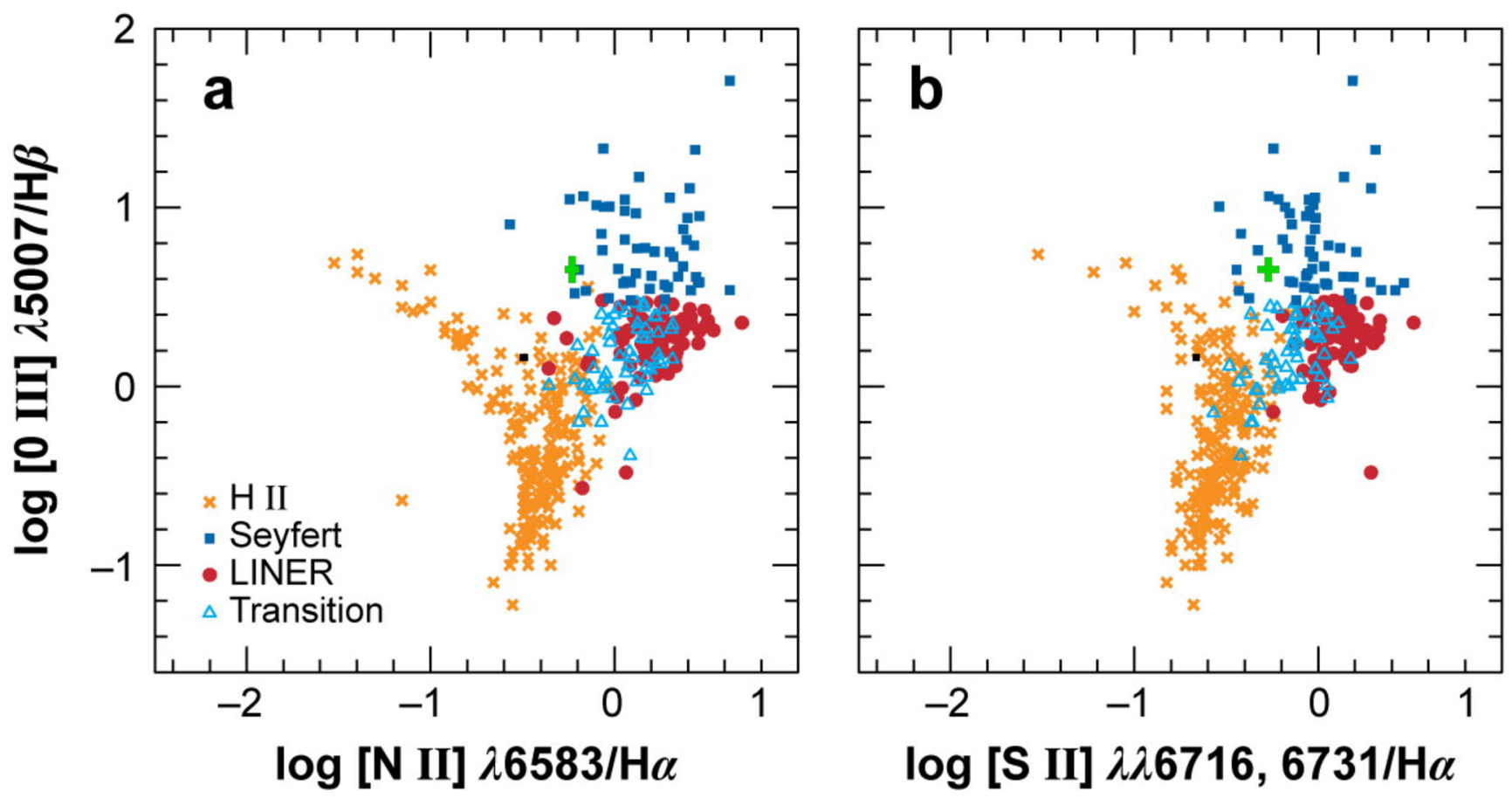

Figure 4. The position and error bars of the line ratios in diagnostic diagrams, indicated by black squares (for the spectrum extracted close to the blue source) and green pluses (for the spectrum extracted at the position of the ULX; figure adapted from Ho 2008). Close to the blue source the line ratios are fully consistent with a $\mathrm{H}$ II region, while at the position of the ULX the line ratios are more like those seen in Seyferts, indicating that the ULX might play a role in ionizing the part of the nebula that surrounds it.

MOLLY and applying hfix, we average them using the task average. We then measure the radial velocity of the emission line region in the UVB and VIS spectra separately by using the mgfit function to fit a set of Gaussian curves with a common velocity offset to the above-mentioned lines. The average radial velocity we find in this way is $351 \pm 4 \mathrm{~km} \mathrm{~s}^{-1}$.

From the fits to the spectra we also obtain line ratios of $\log ([\mathrm{O} \mathrm{III}] / \mathrm{H} \beta), \log ([\mathrm{N}$ II $] / \mathrm{H} \alpha)$ and $\log ([\mathrm{S} \mathrm{II}] / \mathrm{H} \alpha)$. These ratios are plotted in Fig. 4, adapted from Ho (2008). Close to the blue source the line ratios are fully consistent with a $\mathrm{H}$ II region, while at the position of the ULX the line ratios are more like those seen in Seyferts.

The He II $\lambda 4686$ line, which acts as a photon counter for photons in the 54-200 eV band (Pakull \& Angebault 1986), has been observed in several ULX nebulae (cf. Pakull \& Mirioni 2002; Kaaret \& Corbel 2009; Moon et al. 2011; Gutiérrez \& Moon 2014). We do not detect it in our X-shooter spectrum, with a $2 \sigma$ ( 99.7 per cent confidence) upper limit of $8.8 \times 10^{-17} \mathrm{erg} \mathrm{cm}^{-2} \mathrm{~s}^{-1}$ (corresponding to a luminosity of $1.2 \times 10^{35} \mathrm{erg} \mathrm{s}^{-1}$ ).

\subsection{Probability of chance superposition}

J0047 is located close to one of the spiral arms of NGC 253 (see Fig. 5). In such an environment, the probability of a chance superposition of an unrelated object may not be negligible. Using the IRAF task DAOFIND we search for point sources in our VLT/ISAAC $K s$-band image (Heida et al. 2014) that are equally bright or brighter than the apparent magnitude of the candidate RSG $(K s=17.2 \pm 0.5)$. We find that there are 50 such sources in our image, that has a total area of $7200 \mathrm{arcsec}^{2}$. The error circle around the X-ray position of the ULX has a radius of $1.1 \mathrm{arcsec}$, or an area of $3.8 \operatorname{arcsec}^{2}$ (see Heida et al. 2014 for the calculation of the size of the error circle and details of the ISAAC NIR image). This means the probability of finding a source as bright as the RSG in the error circle by chance superposition is 2.6 per cent. However, this image includes part of a spiral arm of NGC 253 while the ULX is located just outside this arm, where the point source density is lower. Therefore, 2.6 per cent is a conservative upper limit. Due to the inhomogeneous distribution of stars, the value for the chance superposition probability depends strongly on the part of the image that is selected for the calculation. Therefore, it is not possible to find an accurate value for the chance superposition probability. With an upper limit of 2.6 per cent we cannot exclude the possibility that the RSG and ULX are unrelated. Only a robust measurement of radial velocity variations will prove whether the RSG is orbiting the compact object responsible for the ULX emission.

\section{DISCUSSION AND CONCLUSIONS}

We obtained low- and medium-resolution $H$ band spectra of the counterpart of J0047, a ULX in NGC 253. From a cross-correlation with spectra of RSGs in the Milky Way, LMC and SMC we conclude that the candidate counterpart is most likely an early M-type supergiant.

In the near-UV and visible parts of our X-shooter spectrum we detect several emission lines with line ratios that are in between those of $\mathrm{H}$ II regions and Seyferts (see Fig. 4). The 2-D profile of the emission lines shows that they are extended in the direction and peak at the position - of a bright and blue optical source at $\sim 5$ arcsec (corresponding to $\sim 85 \mathrm{pc}$ at the distance of NGC 253) from the RSG. This source is not visible in our NIR image but was reported by Bailin et al. (2011) to have $\mathrm{M}_{\mathrm{V}}=-8.1$ and $V-I=0$, 


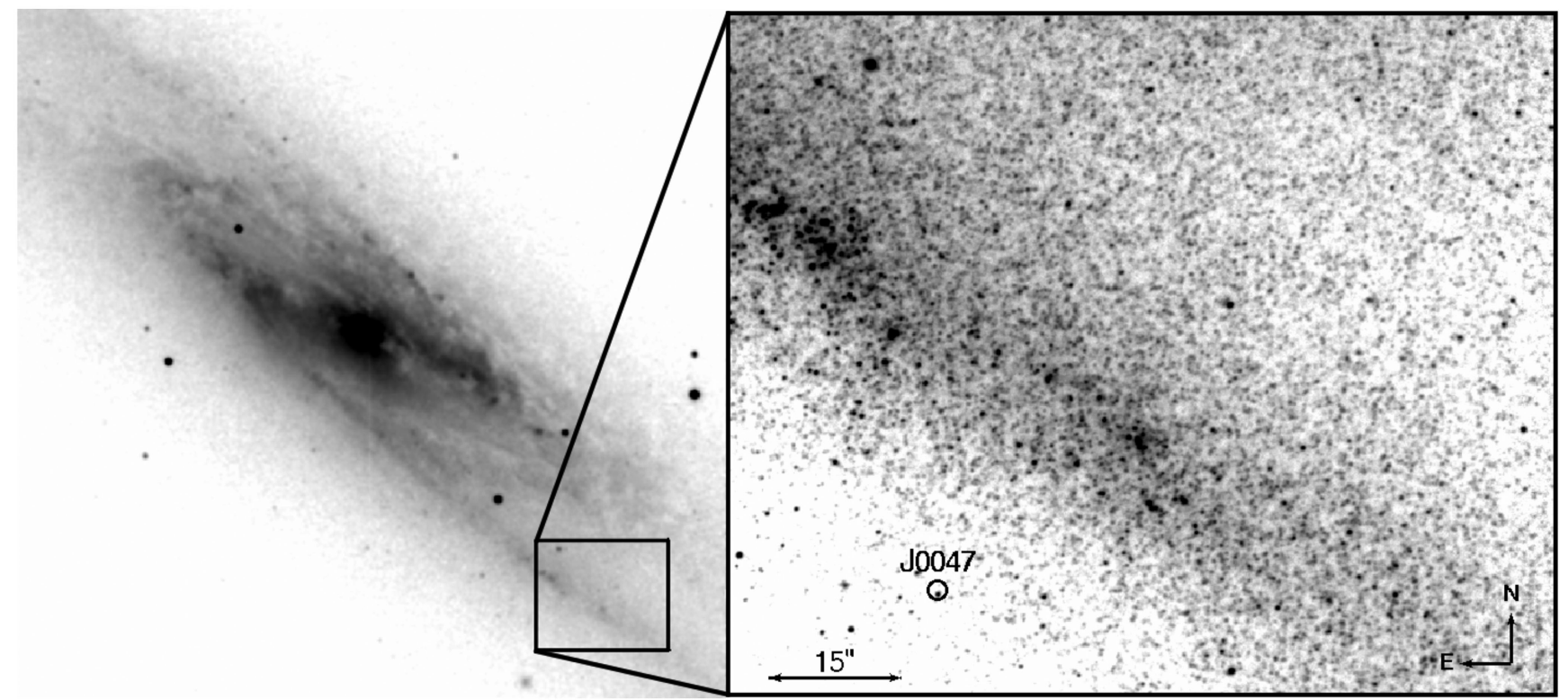

Figure 5. NIR image of the region around J0047. Left: 2MASS image of NGC 253. Right: Our VLT/ISAAC Ks-band image with the X-ray position of the ULX indicated by the black circle (radius 1.1 arcsec, 95 per cent confidence).

consistent with a late B or early A supergiant. Close to this blue source the ratios of the emission lines are different from the ratios found at the position of the ULX, and fully consistent with a $\mathrm{H}$ II region. We do not detect the $\mathrm{He}$ II $\lambda 4686$ line that is indicative of $\mathrm{X}$-ray illuminated nebulae, with a $2 \sigma$ upper limit of $8.8 \times 10^{-17}$ erg $\mathrm{cm}^{-2} \mathrm{~s}^{-1}$ (corresponding to a luminosity of $1.2 \times 10^{35} \mathrm{erg}$ $\mathrm{s}^{-1}$ ). For comparison, Moon et al. (2011) report a luminosity in this line of $\sim 8.8 \times 10^{34} \mathrm{erg} \mathrm{s}^{-1}$ for M81 X-6, a ULX with an X-ray luminosity similar to J0047. We propose that the nebula is excited both by young stars and the ULX, tracing a region with recent star formation where also the RSG was formed. A detection of or a stronger limit on - the $\mathrm{He}$ II $\lambda 4686$ line could (dis)prove this scenario.

We use these lines to measure the projected radial velocity of NGC 253 at the position of the ULX and find a value of $351 \pm 4 \mathrm{~km} \mathrm{~s}^{-1}$. This is compatible with the value of $350 \pm 30 \mathrm{~km} \mathrm{~s}^{-1}$ reported by Hlavacek-Larrondo et al. (2011), who measured the rotation curve of the galaxy using $\mathrm{H} \alpha$ observations. The radial velocity of the counterpart, as measured from our VLT/X-shooter spectrum taken in 2014 August, is $417 \pm 4 \mathrm{~km} \mathrm{~s}^{-1}$. This proves that it is not a foreground or background object but is located in NGC 253, confirming its absolute $K s$-band magnitude of $-10.5 \pm 0.5$ and its identification as an RSG (Heida et al. 2014) This absolute magnitude is too bright for asymptotic giant branch (AGB) stars, that have absolute $K$-band magnitudes between -6.4 and -8.2 (Knapp et al. 2003). This makes J0047 a very strong candidate for a ULX with an RSG donor star. The ultimate proof for this would be the detection of periodic radial velocity variations in the RSG.

With an effective temperature in the range of 3000-3900 K (spectral types M0-3, Tokunaga 2000), the expected radius of the RSG based on its absolute $K s$-band magnitude of -10.5 is $\approx 600-1600$ $\mathrm{R}_{\odot}$. Assuming Roche lobe filling RSG with a mass of $10 \mathrm{M}_{\odot}$, the orbital period expected for such an RSG orbiting a $\mathrm{BH}$ is 4.5 - 20 years. The apparent radial velocity amplitude depends on this period as well as on the mass ratio and the inclination of the system. For instance, for an inclination of $60^{\circ}$, this velocity amplitude varies from $\sim 20 \mathrm{~km} \mathrm{~s}^{-1}$ in the case of a stellar mass $\mathrm{BH}$, to hundreds $\mathrm{km} \mathrm{s}^{-1}$ for an IMBH (see Fig. 6). We do not detect a significant difference in radial velocity between our 2013 June and 2014 August spectra $\left(\Delta v=10 \pm 70 \mathrm{~km} \mathrm{~s}^{-1}\right)$. The large uncertainty is due entirely to the 2013 June low resolution, low S/N MMIRS spectrum, and prevents us from putting strong limits on the mass of a possible $\mathrm{BH}$ companion. We calculate the probability of detecting a velocity shift larger than $80 \mathrm{~km} \mathrm{~s}^{-1}$ (our $1 \sigma$ upper limit on the velocity shift) for a range of $\mathrm{BH}$ masses and orbital periods, taking into account that the orbital phase is unknown. For a system inclination of $60^{\circ}$, we find that if the orbital period is $4.5 \mathrm{yr}$, we have a $<50$ per cent chance of detecting a velocity shift if $\mathrm{M}_{\mathrm{BH}}<100 \mathrm{M}_{\odot}$. If the orbital period is more than 10 years we would not detect $\mathrm{RV}$ variations even if the RSG orbits a $1000 \mathrm{M}_{\odot} \mathrm{BH}$

However, we do measure a radial velocity offset of $66 \pm 6 \mathrm{~km} \mathrm{~s}^{-1}$ between the RSG and its surroundings (as traced by the emission lines). We consider three possible explanations for this offset.

(1) The RSG and the X-ray source are unrelated, and the RSG is a single runaway star. In this scenario the ULX has another, fainter donor star that we do not detect. Eldridge, Langer \& Tout (2011) calculated the runaway fraction of RSGs in a simulated stellar population; at solar metallicity, they find that 7.2 per cent of RSGs have a space velocity $>30 \mathrm{~km} \mathrm{~s}^{-1}$ (their table 4). Of those runaway RSGs, $\sim 10$ percent have a space velocity $>60 \mathrm{~km} \mathrm{~s}^{-1}$ (the $1 \sigma$ lower limit to our velocity offset; their fig. 2). We combine this with the upper limit of 2.6 per cent that we find for the chance superposition probability of the RSG with the ULX. Using these conservative estimates (since we only measure the radial velocity of the RSG and the space velocity is likely larger) we find that the probability of a chance superposition of the ULX with a runaway RSG is $\lesssim 0.02$ per cent.

(2) The RSG is the donor of the ULX and the offset of $66 \pm 6 \mathrm{~km} \mathrm{~s}^{-1}$ is the systemic velocity of the binary: the $\mathrm{BH}$ (or neutron star) received a large natal kick (NK) and dragged the RSG (or its progenitor) with it (Repetto, Davies \& Sigurdsson 2012). Some Galactic BH binaries show high-peculiar velocities (cf. Miller-Jones 


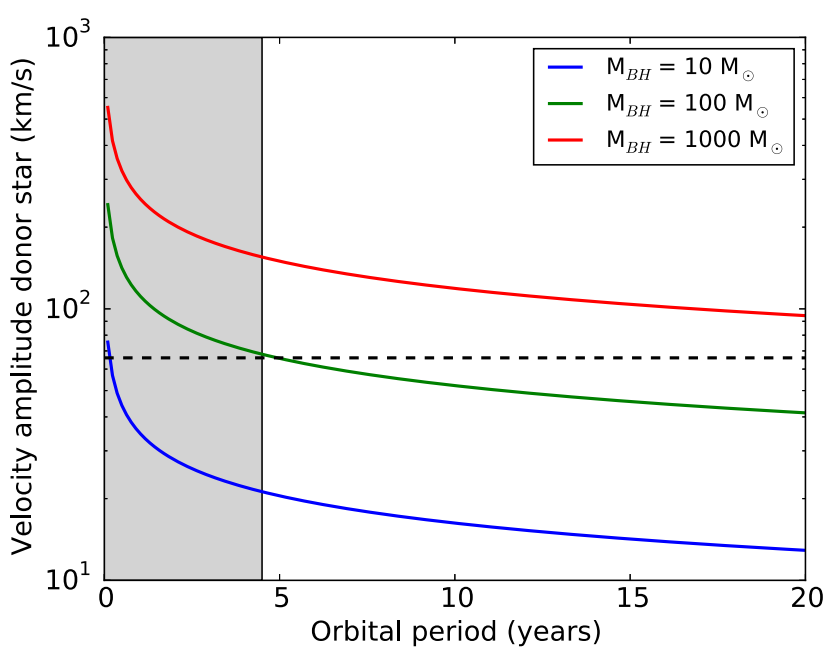

Figure 6. The radial velocity amplitude for a $10 \mathrm{M}_{\odot}$ mass donor in orbit around a 10,100 , or $1000 \mathrm{M}_{\odot} \mathrm{BH}$ (lower, middle and upper line, respectively) as a function of orbital period. A system inclination of $60^{\circ}$ is assumed. Assuming a Roche lobe filling RSG, the expected orbital period is between 4.5 and $20 \mathrm{yr}$. Smaller donor radii give shorter orbital periods; a higher donor mass requires a higher $\mathrm{BH}$ mass to reach the observed velocity. The black dashed line indicates a velocity of $66 \mathrm{~km} \mathrm{~s}^{-1}$; the hashed area is ruled out based on the necessary radius of the RSG.

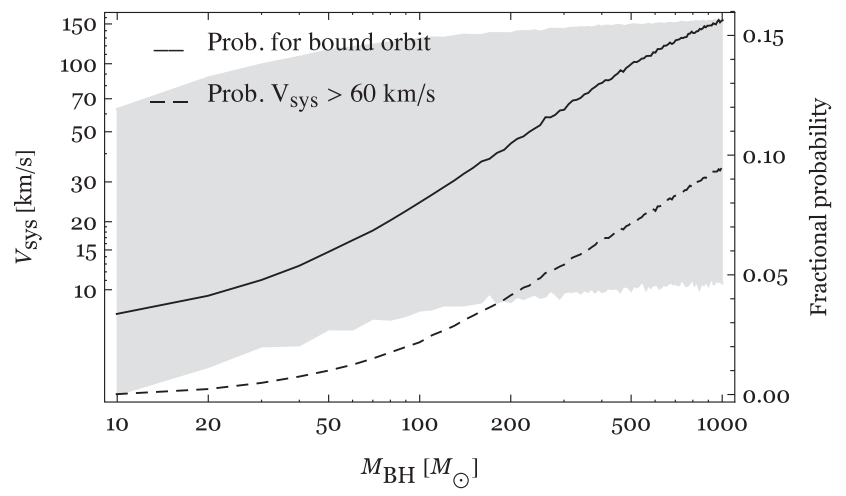

Figure 7. Probability fraction for the binary to stay bound in the BH formation event (solid line), and probability fraction that such a surviving binary acquires a systemic velocity greater then $60 \mathrm{~km} \mathrm{~s}^{-1}$ (dashed line), both as a function of the $\mathrm{BH}$ mass. The shaded area corresponds to the range of possible systemic velocities acquired by the binary as a function of the $\mathrm{BH}$ mass.

2014). However, these are all BHs with low-mass companions. Instead, Cyg X-1, a binary containing a stellar mass BH with an $\sim 30 \mathrm{M}_{\odot}$ donor star (which is of the same magnitude as the RSG mass), has a peculiar velocity of only $\sim 20 \mathrm{~km} \mathrm{~s}^{-1}$. The difficulty with this scenario is that the probability of kicking a $\mathrm{BH} / \mathrm{NS}$ plus $10 \mathrm{M}_{\odot}$ donor in a wide orbit to a high systemic velocity, while keeping the binary intact, is very small.

In order to quantify this we simulate a population of binaries containing the progenitor of the $\mathrm{BH}$ with a $10 \mathrm{M}_{\odot}$ star as companion, calculating the effect of the BH formation event on the orbital and kinematical properties of the binary. The distribution of initial binary separations is taken to be flat between a minimum value $a_{\text {min }}$, corresponding to the orbital separation at which either one of the two components fills its Roche lobe, and a maximum value of $1000 a_{\min }$. We model the $\mathrm{BH}$ formation event assuming a mass equal to half the mass of the $\mathrm{BH}$ is ejected instantaneously from the binary. In the most standard formation scenario, BHs are thought to be produced via fallback on to the nascent neutron stars. In such a scenario, BHs are then expected to receive a NK, as neutron stars do. The NK will be however reduced by the ratio $M_{\mathrm{NS}} / M_{\mathrm{BH}}$, simply by conservation of linear momentum: $V_{\mathrm{NK}, \mathrm{BH}}=V_{\mathrm{NK}, \mathrm{NS}}\left(M_{\mathrm{NS}} / M_{\mathrm{BH}}\right)$, where $V_{\mathrm{NK}}$, NS is drawn from a Maxwellian distribution as in Hobbs et al. (2005). The NK has a random orientation with respect to the binary orbital plane. Through the laws of conservation of energy and orbital angular momentum, we calculate the orbital parameters $(a, e)$ of the binary right after the $\mathrm{BH}$ is formed. We select among those binaries which stay bound in the process, those ones which satisfy two constraints.

The first constraint is that the systemic velocity acquired by the binary in the $\mathrm{BH}$ formation event, i.e. the velocity of the new centre of mass with respect to the old one, has to be larger than $60 \mathrm{~km} \mathrm{~s}^{-1}$. The second constraint comes from the spectral and photometric properties of the companion star. The orbital separation after the $\mathrm{BH}$ is formed has to allow for mass transfer to happen on the nuclear time-scale of the companion when it fills its Roche lobe as an RSG. This is done making sure that the Roche lobe radius of the companion in the circularized orbit is in the interval $600-1600 \mathrm{R}_{\odot}$.

We show the results of this simulation in Fig. 7. The solid line shows the probability for the binary to stay bound in the BH formation event. The dashed line shows the probability for such a surviving binary to acquire a systemic velocity larger than $60 \mathrm{~km} \mathrm{~s}^{-1}$. Both probabilities are shown as a function of the $\mathrm{BH}$ mass. We also show in the plot the range of possible systemic velocities acquired by the surviving binary; the minimum and maximum value for the systemic velocity are calculated at the 99.7 per cent probability. These simulations were done conservatively assuming an RSG of $10 \mathrm{M}_{\odot}$; if the donor star is more massive, the probabilities will be lower.

Projection effects lower the probability even further. The chance that $v_{\text {sys }}$ is pointed within a degree from our line of sight is of the order of $10^{-5}$, so the space velocity of the RSG with respect to its environment is likely larger than what we measure in the radial direction. Bearing in mind that $\mathrm{BH}$ formation is still very uncertain, we also tested a more conservative formation scenario for the $\mathrm{BH}$, in which the $\mathrm{BH}$ does not receive any $\mathrm{NK}$ at birth, and the peculiar velocity at birth is a consequence of the mass ejection only (Blaauw kick, Blaauw 1961). In such a case, none of the surviving binaries acquire a velocity greater than $60 \mathrm{~km} \mathrm{~s}^{-1}$.

These simulations show that if the velocity difference is due to the systemic velocity only, a $\mathrm{BH}$ of $\gtrsim 50 \mathrm{M}_{\odot}$ is necessary to explain the measured offset.

(3) The RSG is the donor of the ULX and (part of) the offset of $66 \mathrm{~km} \mathrm{~s}^{-1}$ is due to the binary motion of the RSG around the $\mathrm{BH}$. It is difficult to explain the offset as the systemic velocity of the binary, but the velocity amplitude of an RSG orbiting a BH can be hundreds $\mathrm{km} \mathrm{s}^{-1}$. This requires a $\mathrm{BH}$ mass of $\gtrsim 100 \mathrm{M}_{\odot}$ for a system inclination $\leq 60^{\circ}$ (see Fig. 6).

Such a high BH mass seems at odds with what is expected based on analysis of the X-ray spectra of this ULX. However, Pintore et al. (2014) suggest this ULX may be viewed at high inclination, which would mean a lower mass BH is sufficient to explain the RV offset (Fig. 6 shows the RV amplitude assuming an inclination of $60^{\circ}$ ). If beaming plays a role in creating the high X-ray luminosities observed in ULXs, a high inclination might explain the relatively low-X-ray luminosity of this source. The combination of these two effects might be enough to reconcile the two mass estimates. 
With the current data we cannot distinguish among these scenarios for J0047. New, high-quality (X-shooter) spectra will allow us to measure radial velocity shifts if they are present. This will allow us to decide whether this ULX is a good target for dynamical measurements of its $\mathrm{BH}$ mass.

\section{ACKNOWLEDGEMENTS}

Observations based on ESO programme 093.D-0256. We thank the anonymous referee for their comments that helped improve the paper. We want to thank Ben Davies for sharing his X-shooter RSG spectra and Tom Marsh for developing MOLLY. This paper uses data products produced by the OIR Telescope Data Center, supported by the Smithsonian Astrophysical Observatory. Based on observations made with ESO Telescopes at the La Silla Paranal Observatory under programme ID 093.D-0256. TPR's contributions were funded as part of the STFC consolidated grant award ST/L00075X/1.

\section{REFERENCES}

Bachetti M. et al., 2013, ApJ, 778, 163

Bachetti M. et al., 2014, Nature, 514, 202

Bailin J., Bell E. F., Chappell S. N., Radburn-Smith D. J., de Jong R. S., 2011, ApJ, 736, 24

Balser D. S., Rood R. T., Bania T. M., Anderson L. D., 2011, ApJ, 738, 27

Barnard R., 2010, MNRAS, 404, 42

Begelman M. C., 2002, ApJ, 568, L97

Blaauw A., 1961, Bull. Astron. Inst. Neth., 15, 265

Chen Y.-P., Trager S. C., Peletier R. F., Lançon A., Vazdekis A., Prugniel P., Silva D. R., Gonneau A., 2014, A\&A, 565, A117

Chilingarian I., Brown W., Fabricant D., McLeod B., Roll J., Szentgyorgyi A., 2013, in Friedel D. N., ed., ASP Conf. Ser. Vol. 475, Astronomical Data Analysis Software and Systems XXII. Astron. Soc. Pac., San Francisco, p. 189

Colbert E. J. M., Mushotzky R. F., 1999, ApJ, 519, 89

Copperwheat C., Cropper M., Soria R., Wu K., 2007, MNRAS, 376, 1407

Dalcanton J. J. et al., 2009, ApJS, 183, 67

Davies B., Kudritzki R.-P., Figer D. F., 2010, MNRAS, 407, 1203

Davies B. et al., 2013, ApJ, 767, 3

Eldridge J. J., Langer N., Tout C. A., 2011, MNRAS, 414, 3501

Farrell S. A., Webb N. A., Barret D., Godet O., Rodrigues J. M., 2009, Nature, 460, 73

Feng H., Soria R., 2011, New Astron Rev., 55, 166

Förster Schreiber N. M., 2000, AJ, 120, 2089

Freudling W., Romaniello M., Bramich D. M., Ballester P., Forchi V., GarcíaDabló C. E., Moehler S., Neeser M. J., 2013, A\&A, 559, A96

Gladstone J. C., Roberts T. P., Done C., 2009, MNRAS, 397, 1836

Grisé F., Kaaret P., Corbel S., Feng H., Cseh D., Tao L., 2012, ApJ, 745, 123
Gutiérrez C. M., Moon D.-S., 2014, ApJ, 797, L7

Heida M. et al., 2014, MNRAS, 442, 1054

Hlavacek-Larrondo J., Carignan C., Daigle O., de Denus-Baillargeon M.-M., Marcelin M., Epinat B., Hernandez O., 2011, MNRAS, 411, 71

Ho L. C., 2008, ARA\&A, 46, 475

Hobbs G., Lorimer D. R., Lyne A. G., Kramer M., 2005, MNRAS, 360, 974

Horne K., 1986, PASP, 98, 609

Jonker P. G., Torres M. A. P., Fabian A. C., Heida M., Miniutti G., Pooley D., 2010, MNRAS, 407, 645

Kaaret P., Corbel S., 2009, ApJ, 697, 950

Knapp G. R., Pourbaix D., Platais I., Jorissen A., 2003, A\&A, 403, 993

Levesque E. M., Massey P., Olsen K. A. G., Plez B., Josselin E., Maeder A., Meynet G., 2005, ApJ, 628, 973

Liu J., Orosz J., Bregman J. N., 2012, ApJ, 745, 89

Liu J.-F., Bregman J. N., Bai Y., Justham S., Crowther P., 2013, Nature, 503, 500

McClintock J. E., Remillard R. A., 1986, ApJ, 308, 110

McLeod B. et al., 2012, PASP, 124, 1318

Mermilliod J. C., Mayor M., Udry S., 2008, A\&A, 485, 303

Mezcua M., Roberts T. P., Lobanov A. P., Sutton A. D., 2015, MNRAS, 448,1893

Miller-Jones J. C. A., 2014, PASA, 31, 16

Moon D.-S., Harrison F. A., Cenko S. B., Shariff J. A., 2011, ApJ, 731, L32

Motch C., Pakull M. W., Soria R., Grisé F., Pietrzyński G., 2014, Nature, 514, 198

Origlia L., Moorwood A. F. M., Oliva E., 1993, A\&A, 280, 536

Pagel B. E. J., Edmunds M. G., Fosbury R. A. E., Webster B. L., 1978, MNRAS, 184, 569

Pakull M. W., Angebault L. P., 1986, Nature, 322, 511

Pakull M. W., Mirioni L., 2002, preprint (arXiv:0202488)

Pasham D. R., Strohmayer T. E., Mushotzky R. F., 2014, Nature, 513, 74

Pintore F., Zampieri L., Wolter A., Belloni T., 2014, MNRAS, 439, 3461

Repetto S., Davies M. B., Sigurdsson S., 2012, MNRAS, 425, 2799

Roberts T. P., Gladstone J. C., Goulding A. D., Swinbank A. M., Ward M. J., Goad M. R., Levan A. J., 2011, Astron. Nachr., 332, 398

Rousselot P., Lidman C., Cuby J.-G., Moreels G., Monnet G., 2000, A\&A, 354,1134

Sutton A. D., Roberts T. P., Walton D. J., Gladstone J. C., Scott A. E., 2012, MNRAS, 423, 1154

Sutton A. D., Roberts T. P., Middleton M. J., 2013, MNRAS, 435, 1758

Sutton A. D., Done C., Roberts T. P., 2014, MNRAS, 444, 2415

Tao L., Kaaret P., Feng H., Grisé F., 2012, ApJ, 750, 110

Tokunaga A. T., 2000, in Cox A. N., ed., Allen's Astrophysical Quantities, 4th edn. Springer-Verlag, New York, p. 143

Vacca W. D., Cushing M. C., Rayner J. T., 2003, PASP, 115, 389

Vernet J. et al., 2011, A\&A, 536, A105

Webster B. L., Smith M. G., 1983, MNRAS, 204, 743

Wik D. R. et al., 2014, ApJ, 797, 79

This paper has been typeset from a $\mathrm{T}_{\mathrm{E}} \mathrm{X} / \mathrm{L} \mathrm{T} \mathrm{E} \mathrm{X}$ file prepared by the author. 